\title{
Advanced Visualization System for Monitoring the ATLAS TDAQ Network in Real-Time
}

S. Batraneanu, D. Campora, B. Martin,

D. Savu, S. Stancu, L. Leahu 


\section{Outline}

Introduction

$>$ ATLAS trigger and data acquisition(TDAQ) system

$>$ Monitoring the ATLAS TDAQ networks

Advanced visualization system for the TDAQ networks

$>$ Design

- Motivation, requirements and challenges

- Hierarchical 3D model and its layout rules

$>$ Implementation

- Client architecture

- Performance optimizations

- Real-time update

- Interaction mechanisms

Conclusions and future work 


\section{ATLAS Trigger and Data}

Acquisition System(TDAQ) Level-1

$>$ Rejection factor: $10^{6}$

$>$ Outstanding accuracy and efficiency

$>3$ networks

- DataCollection(Level-2)

- BackEnd(Level-3)

- Control

- 6 chassis routers, 200 edge switches

- 7000 interfaces, 2000 nodes Level-3

$>$ Demanding performance requirements

Level-2

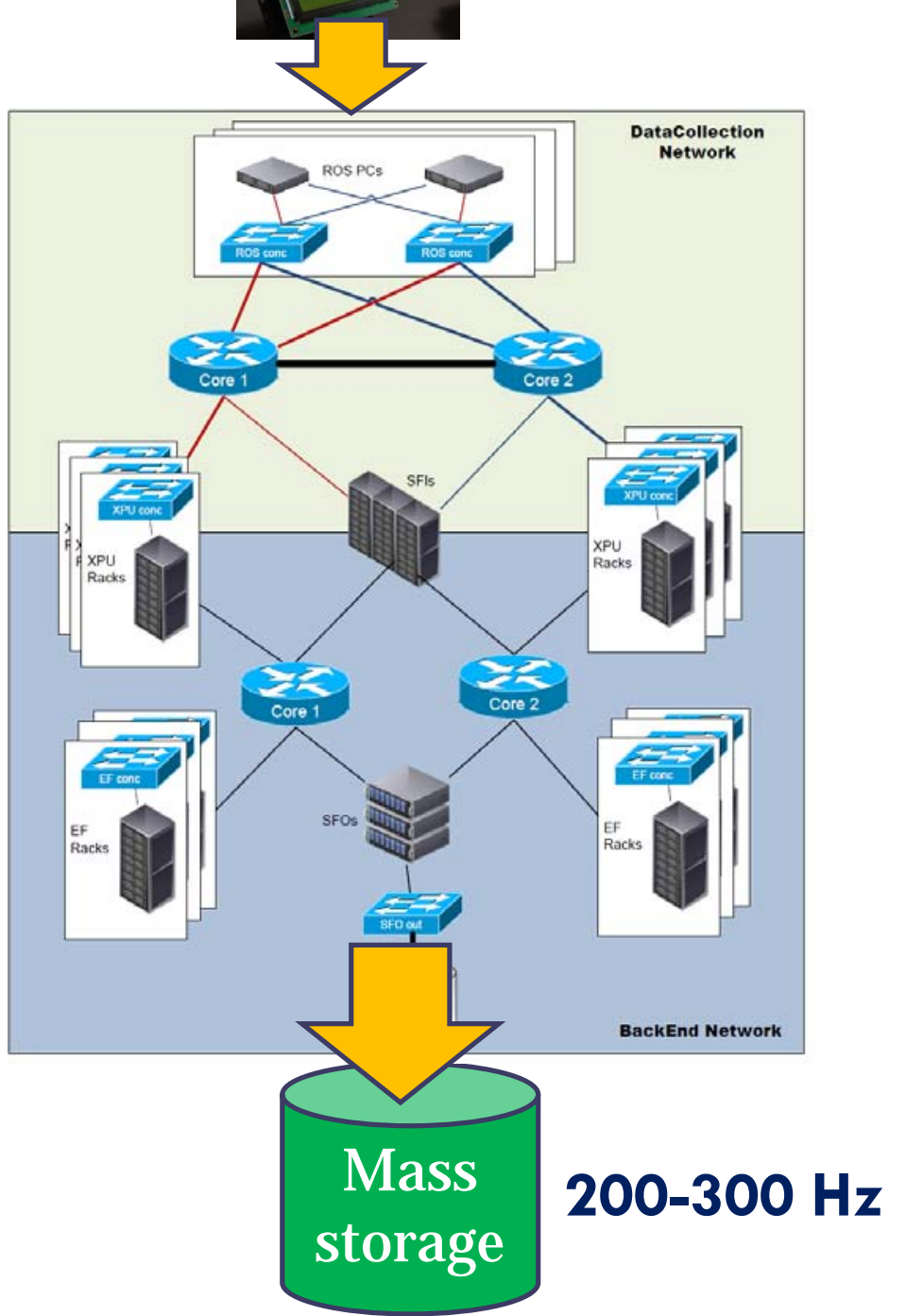




\section{Monitoring software framework}

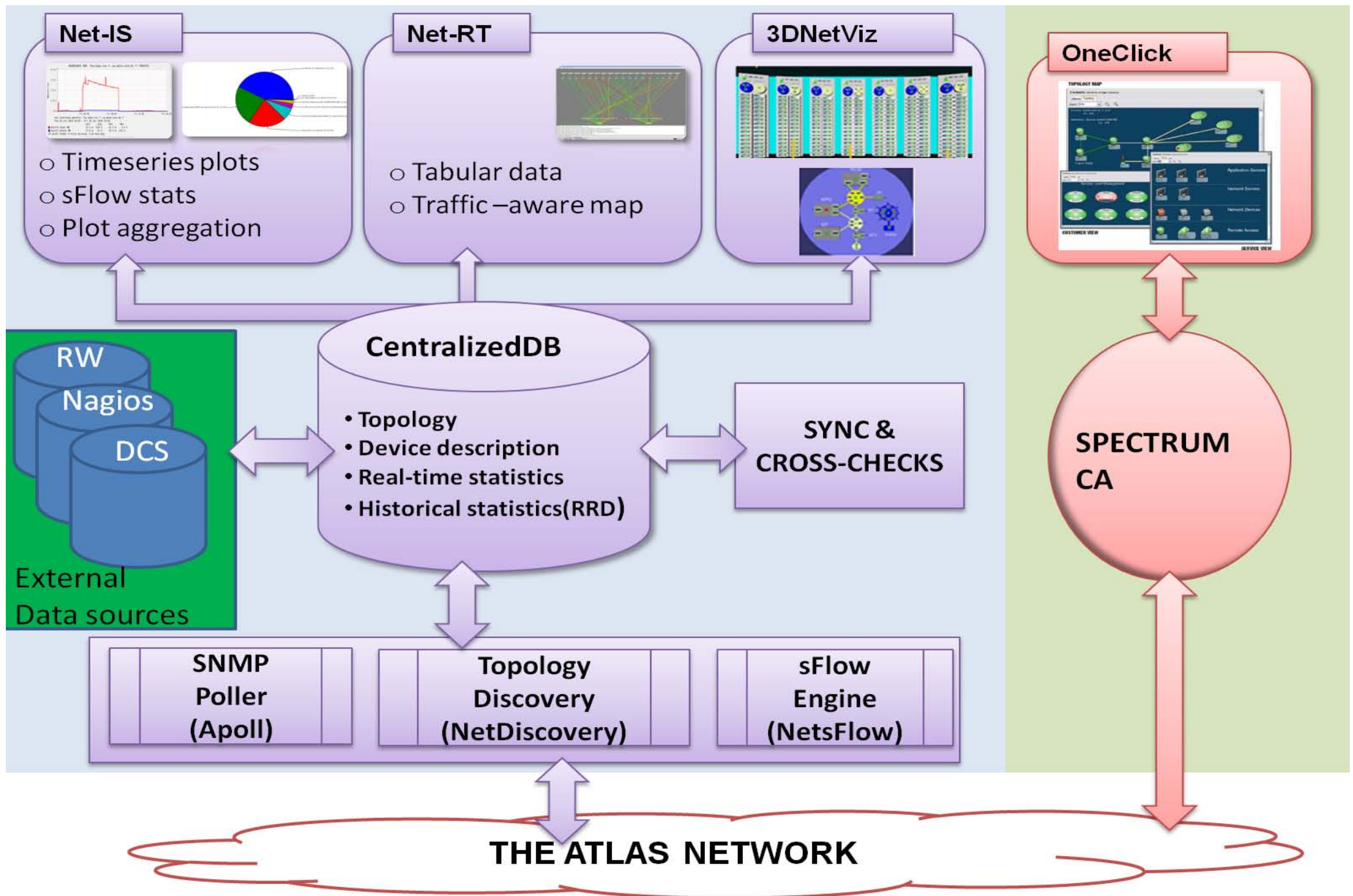




\section{Advanced visualization system -requirements and challenges-}

An efficient visualization system should :

$>$ Be intuitive

$>$ Follow the system's architecture and data flow

$>$ Display the different types of monitoring data in real-time

$>$ Offer the right level of detail

$>$ Provide clear indications regarding the problem

Main implementation challenges

$\square$ Large scale system and overlapping networks

$\square$ Large variable space

$\square$ Real time update (30 seconds)

$\square$ Operation on multiple OSes : Windows, Linux 


\section{D vs. 3D Visualization}

\section{D Visualization}

$>$ Relatively inexpensive in terms of resources and setup

$>$ Visual clutter for overlapping networks

$>$ Two degrees of freedom and restricted navigation paradigms

\section{D Visualization}

$>$ Demanding in terms of processing power, configuration

$>$ Offers additional dimension -> better candidate for large scale complex models

$>$ Six degrees of freedom and natural navigation paradigms (walk, fly)

$>$ Camera-object distance can be evaluated 
Hierarchical

Processing farm 3D Model

Network affiliation - >COlor

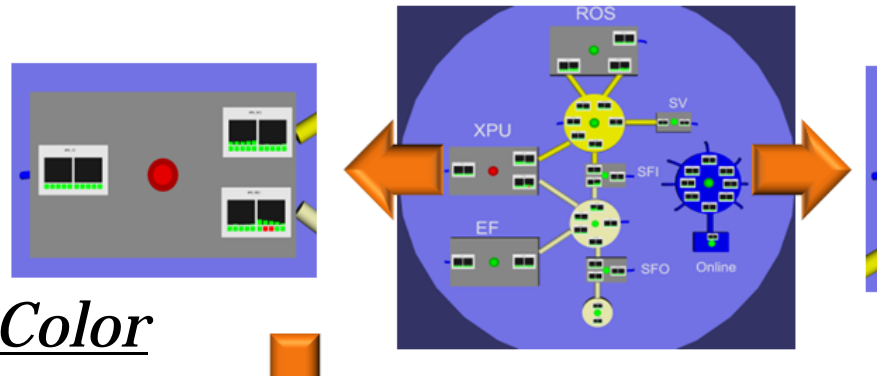

Network core Overall status ->Color

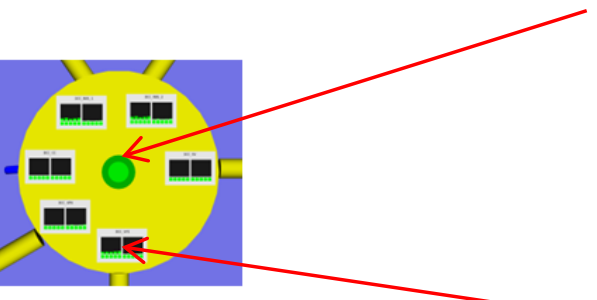

Aggregated traffic panels

Devices

$\circ \cdot \circ \cdot \circ \cdot \circ$

Racks

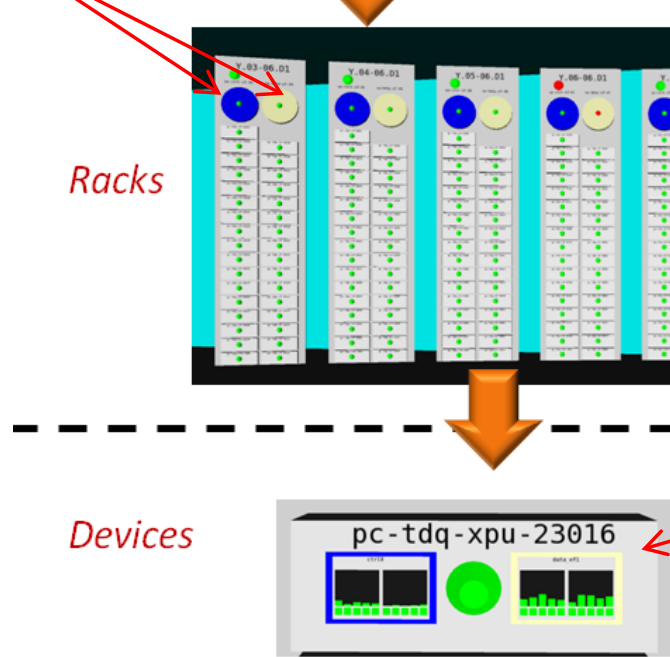

Traffic quantity $->$ Size

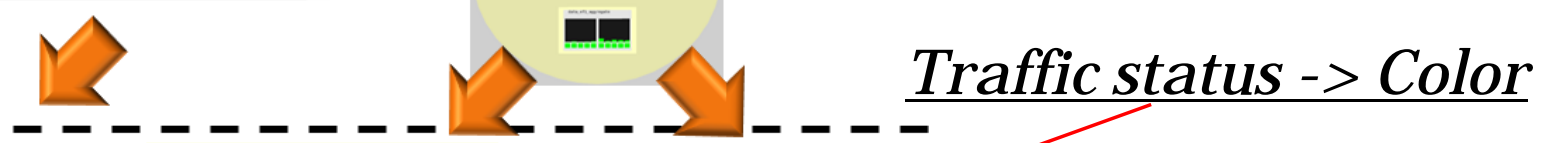

Traffic panels

Data network type->Saturation

Device type - > Shape

data_ef1_aggregate

uplink__w-data-core-dc-02.5/9

Traffic status - > Color 


\section{Optimized object layout}

Top level view

$>$ Follows data flow

$>$ Control network as a backplane

Panoramic rack view

Aspect ratio improvement

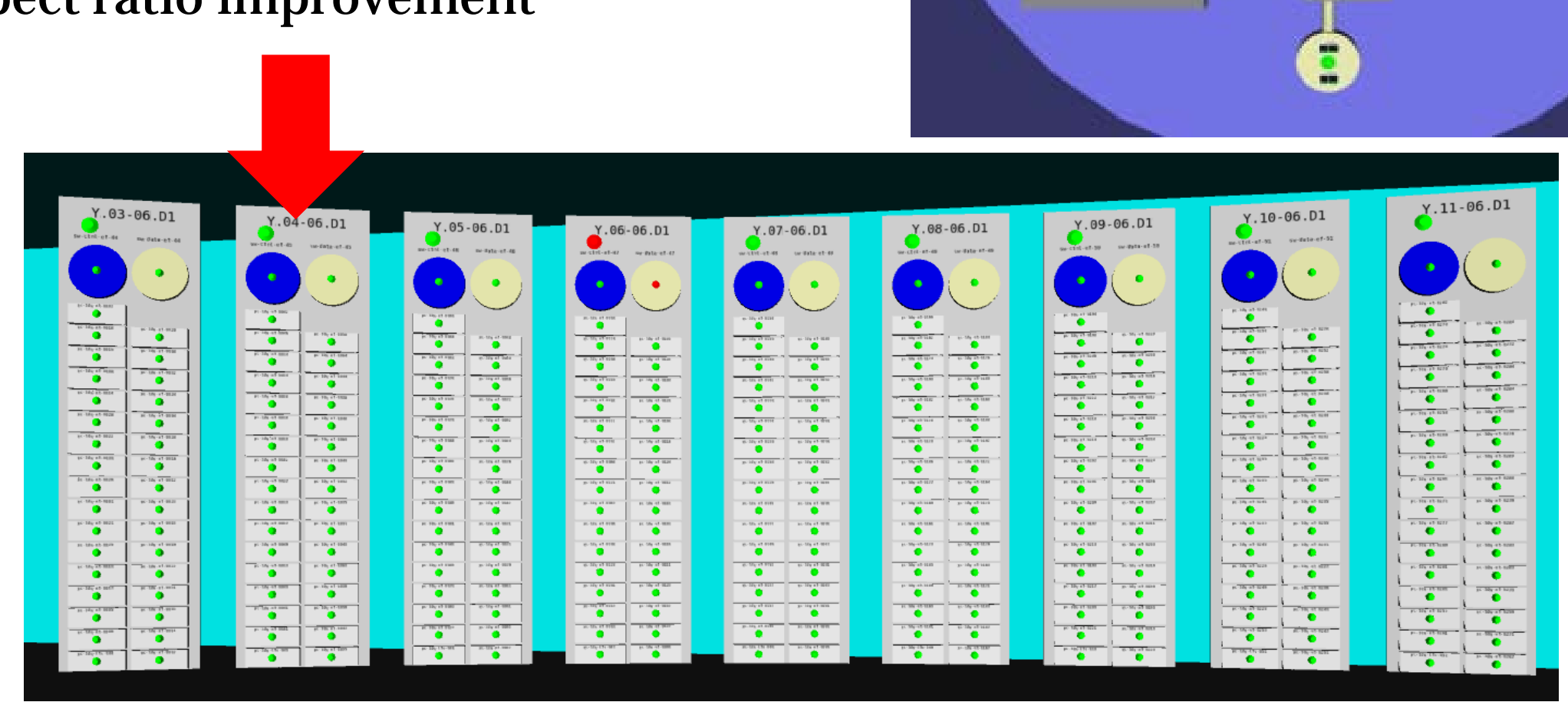




\section{OSG implementation - overview}

\section{OpenSceneGraph(OSG)}

$>$ Open source, portable, high-performance framework based on $\mathrm{C}++$ and OpenGL

> Rigorous structure based on STL and design patterns (visitor, callback)

\section{Why did we choose OSG?}

$>$ Thin wrapper on top of OpenGL -> access to low-level rendering options

$>$ Rendering statistics display and API - > essential for performance tuning

$>$ Bit masks for selection and specialized event handlers for interaction

Profited from the scene creation to perform several optimizations

$>$ Frame rate $>30$ fps

- Minimize overall traversal time: UPDATE+CULL+DRAW

- Adjust LOD ranges

> Real-time update impact minimization 


\section{Client architecture}

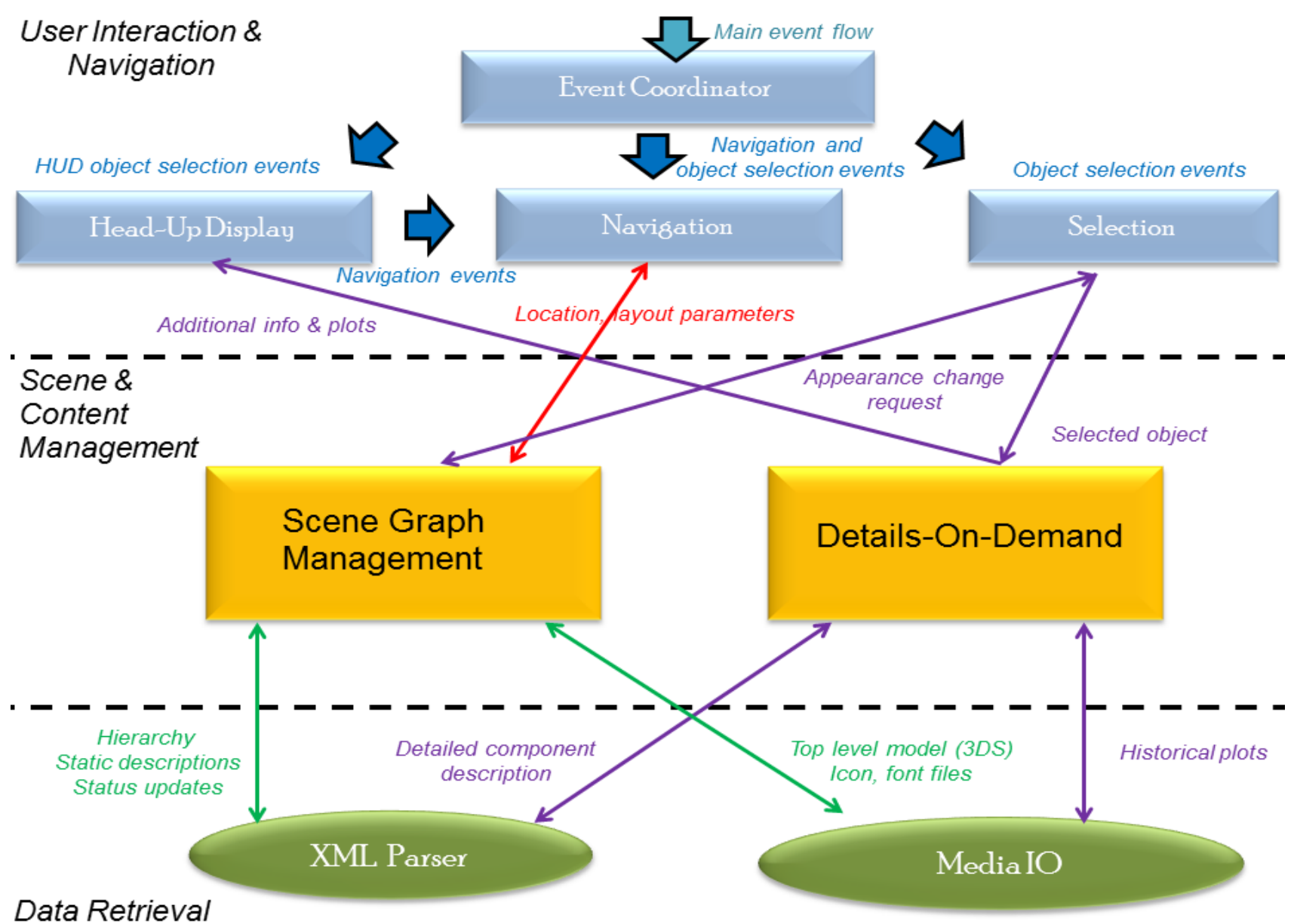




\section{Rendering and scene graph optimizations}

Rendering optimizations

Impacts DRAW traversal time

Geometry rendering - > best solution was to use vertex arrays + triangle primitives + color binding per vertex $->14 \%$ decrease

Custom geometry nodes optimized for fast rendering - > 15\% decrease

Text rendering $->\sim 75 \%$ decrease

Low resolution object versions to use in Level Of Detail

Scene graph restructuring

Impacts CULL traversal time

Eliminated Transform nodes at the panel level-> $-66 \%$ decrease LOD node rearrangement for flexibility 


\section{Real-time update}

Based on visibility and proximity

New targeted update mechanism based on temporal coherence

Tested different granularities

$>$ Individual node - $><30$ fps

$>$ Device node - 26\% increase

$>$ Rack node - 38\% increase

Decreased maximum completion time by spreading the updates over multiple

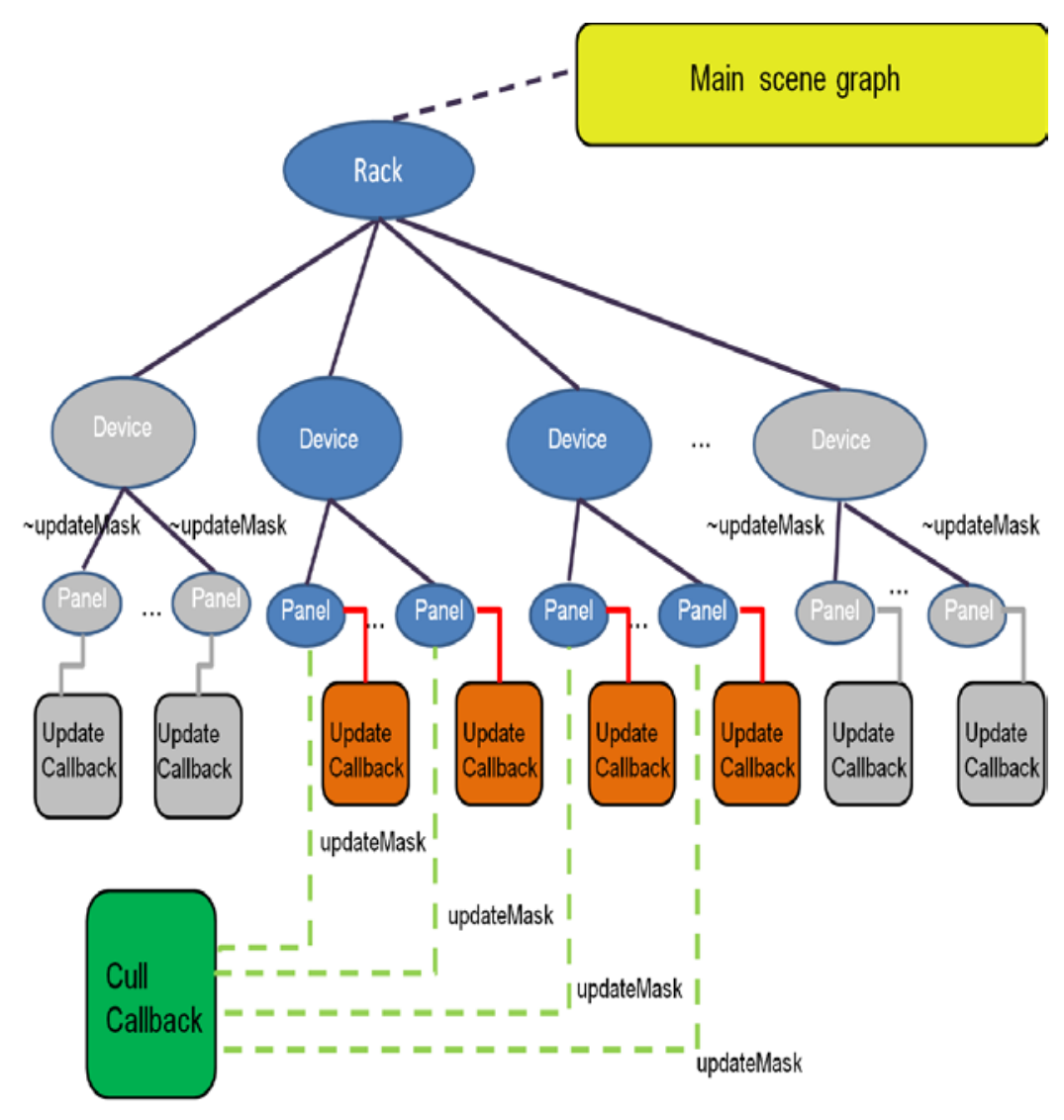
frames 


\section{Interaction mechanisms}

Mixing free and guided navigation

Context aware navigation

> Based on layout parameters

> Different navigation paradigm

$>$ Radial navigation

> Field of view and speed control

Selection and highlight mechanism

Details-On-Demand in a Head-Up Display

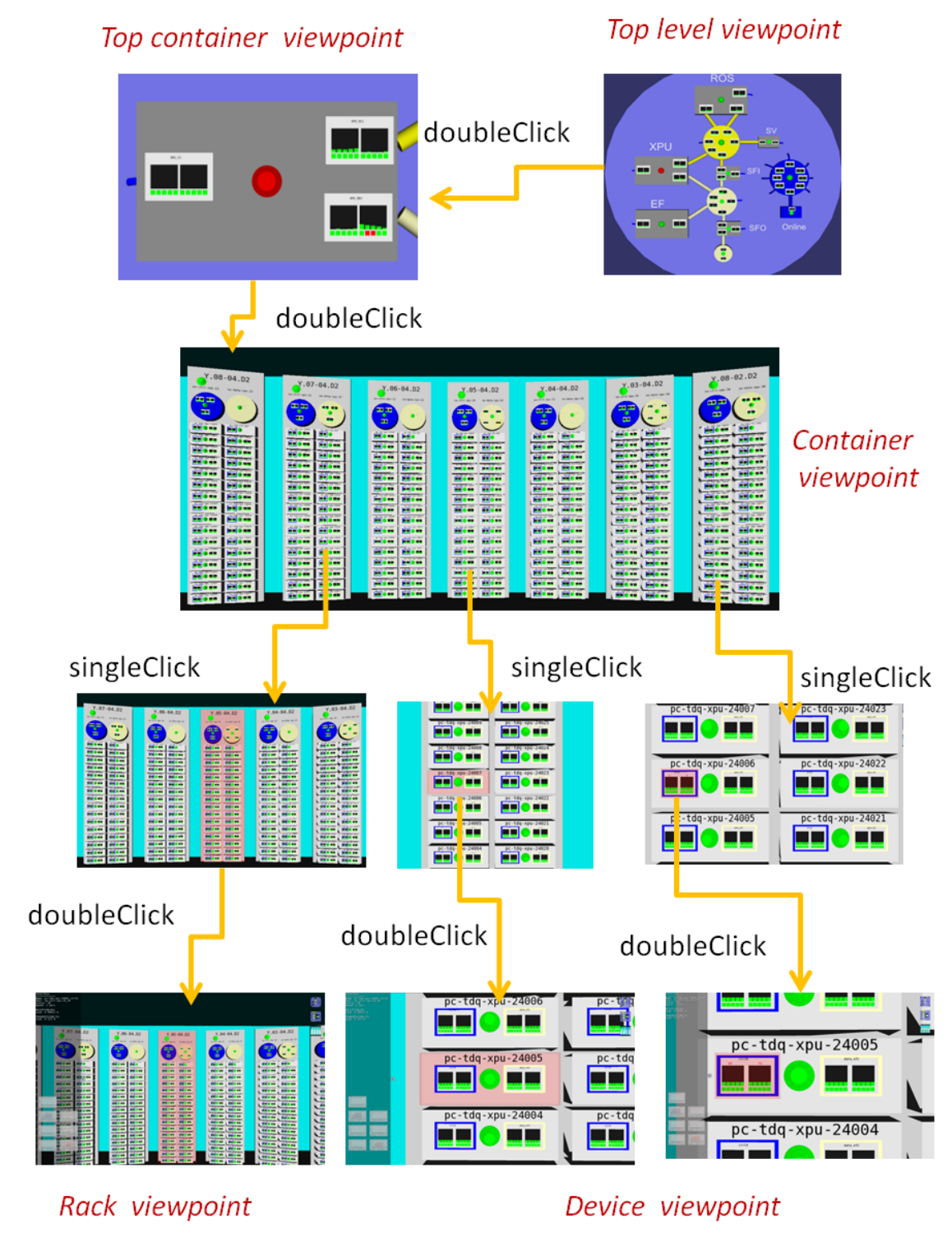




\section{Conclusions and future work}

$>$ Identified specific visualization requirements

$>$ Chose $3 \mathrm{D}$ visualization and InfoViz guidelines

$>$ Used open-source low-level framework OSG

$>$ Intuitive interaction and navigation

$>$ Frame rate $>30 \mathrm{fps}$

Future work

$>$ Integration of data taking parameters

$>$ Rule-based expert system to improve error propagation rules

$>$ Multiple views 